\title{
A memory boost for the elderly
}

Nat. Neurosci. https://doi.org/10.1038/s41593-019-0371-x

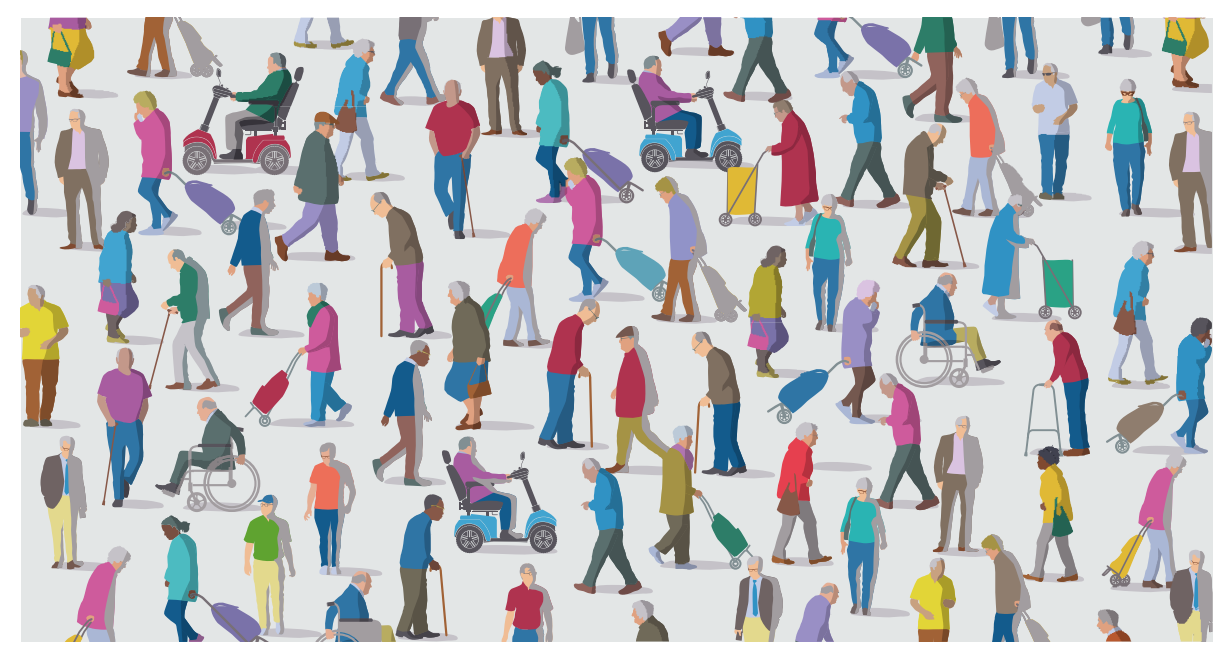

Credit: smartboy10 / DigitalVision Vectors / Getty

Working memory declines with age. Understanding why that happens and whether it can be reversed is increasingly important for the world's ageing population.

A new study by Robert M. G. Reinhart and John A. Nguyen of Boston University uses electroencephalography (EEG) and high-definition transcranial alternatingcurrent stimulation (HD-tACS) to determine the neural bases and reversibility of working memory decline in healthy 60-75 year-olds. The authors find that brain rhythm coupling in temporal cortex (associated with working memory maintenance) and phase synchronization in frontotemporal cortex (associated with information integration) are impaired in older adults. However, $25 \mathrm{~min}$ of stimulation tailored to individual participants significantly improved both neuronal communication and accuracy in a working memory task-so much so that older adults were indistinguishable from 20-year-old controls.

This study provides causal evidence for the role of frontotemporal intra- and interregional functional connectivity in working memory decline. It also suggests that that this decline is not irreversible, providing important initial evidence for future clinical interventions.

\section{Stavroula Kousta}

Published online: 2 May 2019

https://doi.org/10.1038/s41562-019-0608-4 